\title{
VISUALIZAÇÃO DE ONTOLOGIAS: PERSPECTIVAS PARA A REPRESENTAÇÃO DE DOMÍNIOS MODELIZADOS
}

\author{
ONTOLOGY VISUALIZATION: PERSPECTIVES FOR \\ THE REPRESENTATION OF MODELING DOMAIN
}

\author{
Tainá Reglya \\ Maria Luiza de Almeida Campos Correiob
}

\begin{abstract}
RESUMO
Objetivo: identificar as técnicas de visualização da informação aptas a representar esse Sistema de Organização do Conhecimento. Metodologia: uma abordagem qualitativa, de natureza aplicada e objetivo exploratório onde foi realizado um estudo bibliográfico. Resultados: apresenta e exemplifica as técnicas e ferramentas lista identada, árvores de links e nós, visualização zoomable, preenchimento de espaço, foco mais contexto e paisagem 3D. Conclusões: aponta a inexistência de uma técnica apropriada a todos os cenários onde uma visualização de ontologia pode estar inserida.
\end{abstract}

Descritores: Ontologias. Visualização da informação (contribuição). Visualização de ontologias.

\section{INTRODUÇÃO}

As ontologias de domínio podem ser consideradas um Sistema de Organização do Conhecimento (SOC) e uma de suas funções é de organizar e representar um dado domínio do conhecimento. As ontologias fornecem conceituações compartilhadas sobre determinadas áreas que são passíveis de compreensão tanto por pessoas quanto por máquinas (GRUBER, 1995).

Os objetos de uma ontologia de domínio são representações das

\footnotetext{
a Doutoranda no Programa de Pós-graduação em Ciência da Informação pelo Instituto Brasileiro de Informação em Ciência e Tecnologia (IBICT) e Universidade Federal do Rio de Janeiro (UFRJ). Assistente de Pesquisa no Instituto Brasileiro de Informação em Ciência e Tecnologia (IBICT). E-mail: tainaregly@hotmail.com.

b Doutora em Ciência da Informação pelo Instituto Brasileiro de Informação em Ciência e Tecnologia (IBICT) e Universidade Federal do Rio de Janeiro (UFRJ). Docente do Programa de Pós-Graduação em Ciência da Informação da Universidade Federal Fluminense (UFF) e da Universidade Federal da Bahia (UFBA). E-mail: marialuizalmeida@gmail.com.
} 
entidades como elas existem na realidade (CAMPOS, 2004). Essa representação é construída através de definições que descrevem e representam os elementos de um dado campo do conhecimento, constituindo assim, um modelo conceitual. que pode representar uma área canônica ou de missão ou problema, como por exemplo: Medicina, COVID, Saúde da Mulher, entre outras.

Outro aspecto importante a ressaltar é que uma ontologia "é definida como uma especificação formal e explícita de um conceitualização compartilhada. Fornece uma compreensão comum de um domínio que pode ser comunicada a pessoas e sistemas." (DING, 2002 apud MARCONDES; CAMPOS, 2008). Podendo ser considerada uma abstração, uma visão simplificada do mundo, que tem o objetivo de auxiliar a compreensão, o compartilhamento e o consenso de determinada área do conhecimento (CAMPOS, 2010).

Ao nos deslocarmos a um cenário em que há um grupo de pessoas trabalhando em um mesmo domínio, se torna essencial a organização de conceitos previamente acordados e definidos na formação de uma linguagem que será a base de um sistema de recuperação da informação eficaz. No entanto, devido à complexidade dos domínios do conhecimento, as ontologias podem conter milhares de atributos, como classes e instâncias, tornando difícil a compreensão das relações entre seus conceitos (MENDES NETTO; LIMA, 2017). A utilização de representações gráficas que expressam relações entre os conceitos de um domínio torna mais eficiente o processo de recuperação da informação pelo usuário, além de servir como ferramenta para auxiliá-los na compreensão do domínio representado pelas ontologias.

Nesse cenário, a visualização da informação emerge como disciplina e recurso essencial para auxílio na compreensão de grandes montantes de dados, facilitando assim, o processo de análise do significado contido naqueles dados. A visualização da informação tem o objetivo tornar uma interface amigável para o usuário e de representar graficamente as informações de determinado domínio, de forma que os prejuízos ocasionados por dificuldades na navegação sejam minimizados. Ao ser definida por Mendes Netto, Lima e Pierozzi Junior (2016), a visualização da informação é tida como processo de transformação de dados abstratos em gráficos ou imagens com o objetivo de explorar a capacidade 
de percepção humana para que, a partir das relações espaciais exibidas, se interprete e compreenda as informações apresentadas e, ainda, se deduza novos conhecimentos.

Com o objetivo de identificar as técnicas de visualização da informação aplicáveis a ontologias de domínio, este trabalho tem como metodologia uma abordagem qualitativa, de natureza aplicada e objetivo exploratório. A pesquisa consistiu num estudo bibliográfico desenvolvido a partir de material já elaborado, constituído principalmente de livros e artigos científicos.

\section{VISUALIZAÇÃO DA INFORMAÇÃO}

A utilização de interfaces gráficas começou em meados do século XX e, atualmente, por meio da computação gráfica, é possível construir interfaces ainda melhores a partir do auxílio de recursos gráficos como cores, efeitos, brilho, luminosidade, botões, entre outros (DIAS; CARVALHO, 2007). A expansão da tecnologia e a modernização das formas de comunicação permitiram que fossem disponibilizados crescentes volumes de dados para quem busca e necessita de informação. Entretanto, conforme Freitas et al. (2001), muitas dessas informações podem ser irrelevantes para o objetivo da tarefa realizada, causando sobrecarga de informações para o usuário do sistema. Por isso, necessitamos de métodos efetivos que nos permitam perpassar e manipular essas informações de modo que elas nos ajudem na tomada de decisões, por exemplo (MAZZA, 2009).

$A$ visualização da informação se tornou um tema com desenvolvimento e pesquisa significativos graças ao auxílio do computador para o entendimento do processo visual. Os avanços na área são estimulados pelo aumento no poder e disponibilidade de computadores e pelo avanço das comunicações, como é o caso do World Wide Web, que aumentou a quantidade de dados disponíveis num curto período de tempo (CARD; MACKINLAY, 1997).

Com isso, a visualização se transformou em um recurso inovador para que pessoas consigam encontrar a informação de que precisam de maneira intuitiva e efetiva. Sua utilização ganha evidência na medida em que é reconhecido seu potencial no desenvolvimento de técnicas que apresentem 
dados de forma gráfica. Uma representação eficiente maximiza o aproveitamento cognitivo humano, além de permitir que os usuários encontrem com mais facilidade padrões e agrupamentos de dados organizados e possibilita a descoberta de características camufladas em um grande volume de dados (SHNEIDERMAN, 1996).

À vista disso, a visualização da informação é um novo campo de pesquisa que foca no uso de técnicas de visualização para ajudar pessoas a entender e analisar dados (KERREN et al., 2008). Sua aplicação à recuperação da informação pode transformar o processo de encontrar informações em uma tarefa simples e intuitiva, pois não exige conhecimento técnico complexo e nem grande esforço cognitivo para o usuário. Zhang (2008) afirma que essas representações devem suportar ações como análise de dados, exploração de informações, previsão de tendências, detecção de padrões, descoberta de ritmos, entre outros.

Sem o auxílio da visualização na busca por informação há menos percepção e/ou compreensão dos dados expostos. "[...] a visualização de informações permite a apresentação de dados em formas gráficas de modo que - usuário possa utilizar sua percepção visual para melhor analisar e compreender as informações" (FREITAS et al., 2001, p. 144). Para Card e Mackinlay (1997), uma representação visual consiste em um conjunto de marcas (pontos, linhas, áreas, superfícies ou volumes), suas propriedades retinianas (cor e tamanho) e sua posição no espaço e no tempo.

Os seres humanos têm habilidades perceptivas que são subutilizadas quando pensamos em dispositivos visuais. Usuários podem detectar alterações no tamanho, cor, forma, movimento ou textura, bem como podem reconhecer e recuperar imagens rapidamente (SHNEIDERMAN, 1996). A visualização da informação potencializa o uso de interfaces gráficas ao colaborar nas formas de interação e na apreensão dos dados disponibilizados, pois estimula a cognição e a percepção de seu usuário de modo que ele descubra novas informações a partir da interação com o que vê.

Card, Mackinlay e Shneiderman (1999, p. 7, tradução nossa) relatam que "a visualização da informação é o uso de representações visuais computacionais 
e interativas de dados abstratos utilizadas para ampliar a cognição."c $A$ visualização da informação busca melhorar a interface de interação para que o olhar flua entre os dados expostos com o menor esforço cognitivo possível. Além disso, esses dispositivos gráficos têm o papel de estimular a cognição e a percepção de seu usuário de modo que ele descubra novas informações a partir da interação com o que vê. Em sua pesquisa, Ware (2004) apresenta cinco vantagens proporcionadas pela utilização eficiente de visualizações:

- A compreensão de grandes quantidades de informação pelo usuário;

- A percepção de propriedades de um dado que não podem ser antecipadas através de um padrão que pode ser a base de um novo insight;

- Permite que problemas com dados se tornem imediatamente aparentes, pois faz revelações não apenas dos dados em si, mas também sobre a maneira como são coletados;

- Facilita a compreensão de recursos de grande escala e de pequena escala dos dados;

- E facilita a formação de hipóteses pelo usuário da visualização.

A visualização da informação é um campo de grande utilidade para a Ciência da Informação por agregar técnicas que facilitam o entendimento de informações a partir da transformação de dados abstratos em representações gráficas que potencializam o acesso, a localização e a recuperação da informação organizada (NASCIMENTO; FERREIRA, 2005; VIEIRA; PINHO, 2015). Podemos aplicar a visualização da informação em sistemas de recuperação de informações e, consequentemente, transformar o processo de busca em uma atividade mais simples e intuitiva para seu usuário, visto que a interface não exigiria muito esforço cognitivo e conhecimentos específicos para manipulação. Para tal dispomos de uma gama de técnicas de visualização da informação que são utilizadas para solucionar problemas oriundos de excesso de informações e "[...] também podem ser utilizadas para filtrar, analisar e gerenciar grandes quantidades de dados, como as informações que são geradas

\footnotetext{
c "Information visualization is the use of computer-supported, interactive, visual representations of abstract data to amplify cognition." (CARD; MACKINLAY; SHNEIDERMAN, 1999, p. 7).
} 
e disponibilizadas diariamente na Internet." (NASCIMENTO; FERREIRA, 2005, p. 1266).

Além disso, uma técnica de visualização da informação busca representar de forma gráfica os dados de determinado domínio do conhecimento de forma que a representação gerada desenvolva a capacidade perceptiva de seu usuário para que então, este interprete e compreenda as informações apresentadas de modo que seja capaz de inferir novos conhecimentos a partir da estrutura visual formulada (FREITAS et al., 2001).

Shneiderman (1996) descreveu sete tarefas que podem ser aplicadas às técnicas de visualização da informação. Essas tarefas tornam a representação gráfica mais interativa e possibilitam que seus usuários obtenham mais inferências sobre os dados manipulados. São elas:

- visão geral - ganha visão geral de todos os dados da coleção através da utilização do zoom para expor a coleção inteira;

- zoom - permite ampliar os itens dos quais se tem interesse;

- filtro - retira os as informações que não são relevantes para o usuário;

- detalhe por demanda - possibilita a seleção de um item ou um grupo de itens e a obtenção de detalhes quando solicitado;

- relacionar - possibilita a observação do relacionamento entre os itens;

- histórico - mantém um histórico de ações que permite desfazer, refazer e refinar progressivamente;

- extração - possibilita a extração de subcoleções e parâmetros da consulta realizada pelo usuário.

A partir dessas tarefas, os usuários podem manipular visualizações de modo a gerar novas perspectivas e, consequentemente, diferentes informações. O recurso da interação promove aumento na capacidade exploratória da representação gráfica e permite que o usuário encontre dados e conceitos que, antes, estavam camuflados e não eram de simples assimilação.

Segundo Chen, Härdle e Unwin (2008), a visualização da informação possui dois aspectos fundamentais: a modelagem estrutural e as representações 
gráficas. A modelagem se refere à detecção, extração e simplificação de conceitos e suas relações em dado domínio. Essas relações formam a estrutura que representará conteúdo modelado e dará origem a uma linguagem para representar o domínio selecionado. No contexto do trabalho, a linguagem que enfatizaremos consiste na ontologia, que possui as definições como ponto chave para a modelagem conceitual.

\subsection{VisualizaÇÃo de ONTOLOGIAS}

Podemos considerar as formas de representação gráfica como um dos métodos que nos permitem ter melhor acesso à informação. As representações visuais nos auxiliam a ilustrar conceitos que, se tivéssemos os expressado verbalmente, teríamos dificuldades em explicá-los claramente ao nosso ouvinte (MAZZA, 2009).

Uma visualização exequível a ontologias deve representar de forma gráfica os elementos formadores de ontologias, tais como as classes, relações, axiomas e instâncias de modo que seja possível realizar as tarefas de interação. O uso de visualizações para contemplar ontologias apresenta diversas vantagens como a melhoria da execução de tarefas relativas à ao entendimento do conhecimento implícito e ao alinhamento da informação com seus domínios de aplicação (MENDES NETTO; LIMA, 2017).

Considerando isso, acredita-se que as técnicas de visualização de informação possam ser utilizadas na proposta de uma ferramenta para auxiliar os na compreensão do domínio representado pelas ontologias. No entanto [...] existe uma complexidade na escolha da técnica mais adequada para representar uma ontologia, visto que não existe uma técnica que seja apropriada para todos os tipos de ontologias, bem como para os diferentes tipos de usuários e necessidades informacionais. (MENDES NETTO; LIMA; PIEROZZI JUNIOR, 2016).

Katifori et al. (2007) analisaram cerca de trinta e quatro ferramentas e técnicas de visualização e as sistematizaram em um quadro com questões pertinentes à representação de ontologias e sinalizaram as que já foram utilizadas para representar uma ontologia. Nessa sistematização, os autores categorizam as ferramentas e técnicas de visualização da informação de acordo 
com as características de apresentação de cada técnica, sua possibilidade de interação, funcionalidade e dimensões. As técnicas e ferramentas apresentadas podem ser consideradas como pertencentes a mais de uma categoria. Nesses casos, os autores optaram por considerar os recursos de funcionalidade predominantes para alocar as técnicas e ferramentas plenamente.

Conforme Mendes Netto (2017), a multiplicidade de ferramentas e modelos passíveis de representar ontologias de maneira gráfica torna o processo de escolha sobre qual utilizar um obstáculo para os usuários. Para tal, apresentaremos as categorias elencadas por Katifori et al. (2007) e listaremos em cada categoria as técnicas e ferramentas aplicáveis a ontologias:

- Lista identada - exibe a taxonomia de uma ontologia através de relações de herança representadas como a visualização de uma árvore semelhante à organização de pastas do Windows Explorer. Como exemplo, temos a taxonomia apresentada no software Protégé, utilizado na elaboração de ontologias. Técnica e/ou ferramenta aplicadas à ontologia: Navegador clássico do Protégé (figura 1).

\section{Figura 1 - Visualização do navegador clássico do Protégé na categoria lista} identada

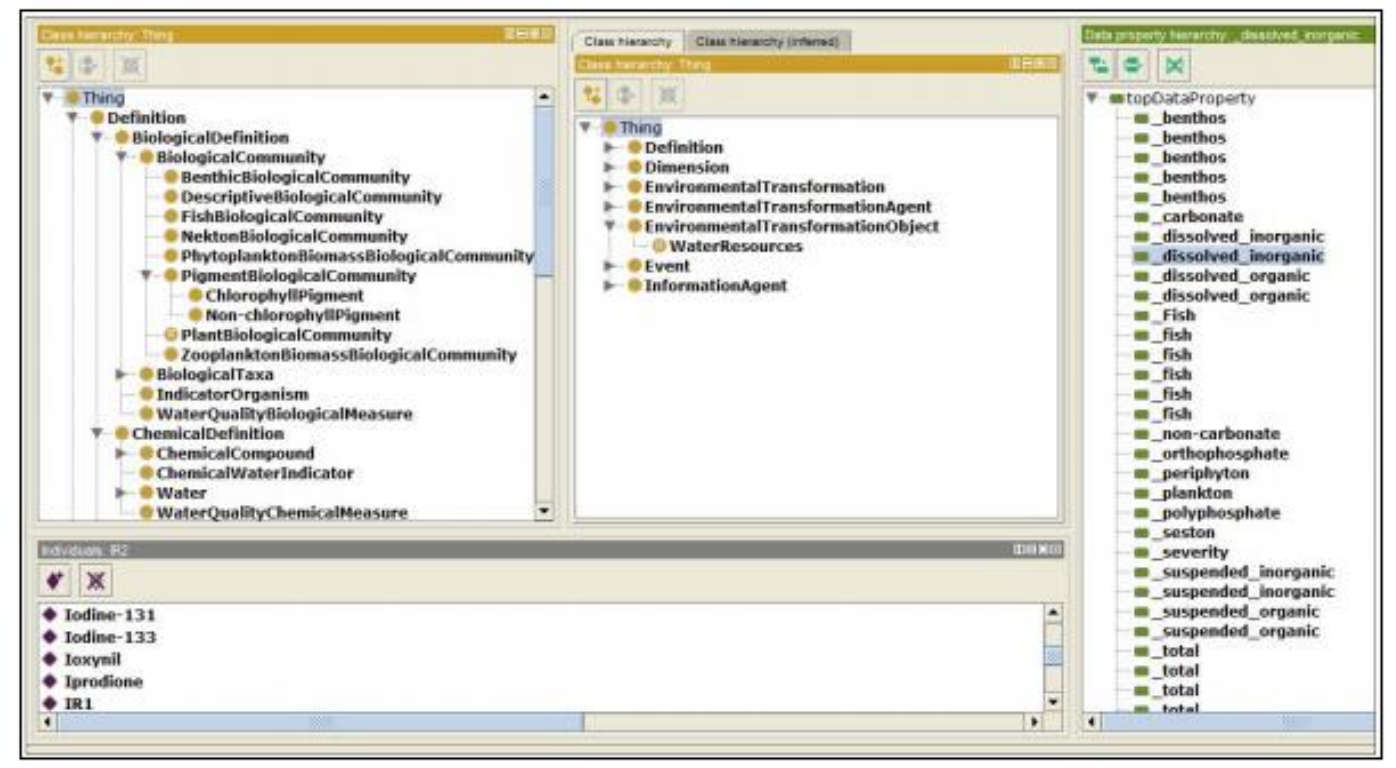

Fonte: Mendes Netto (2017)

- Árvore de links e nós - representa a ontologia como um conjunto de nós interligados apresentando uma taxonomia, com um layout diferente 0 
utilizado na lista identada, onde os nós podem ser expandidos e retraídos pelo usuário para realizar ajustes na exposição de informações relevantes. Esse recurso pode ser apresentado em duas ou três dimensões (figura 2) e podem ser modificadas dinamicamente de acordo com os filtros selecionados. Técnicas e/ou ferramentas aplicadas à ontologia: Navegador clássico do Protégé.

Figura 2 - Visualização da categoria de árvore de links e nós em 3D

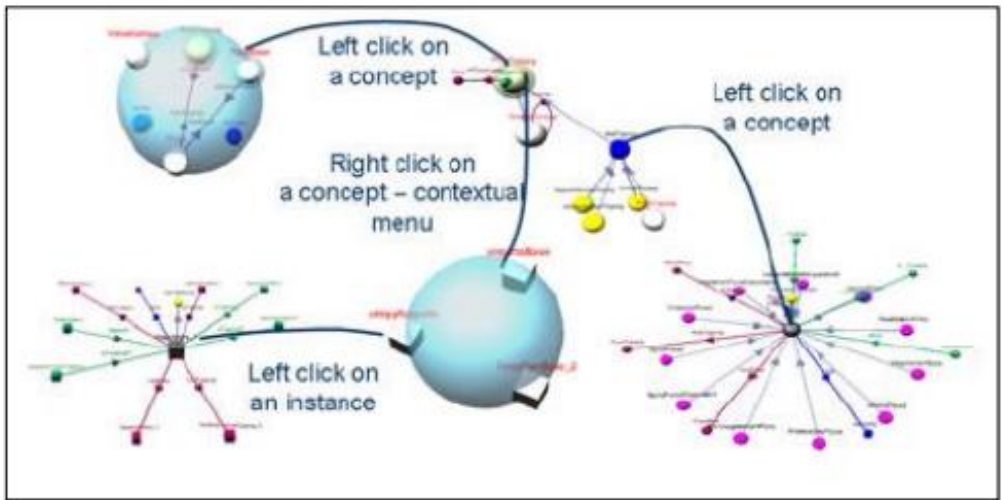

Fonte: Netto (2017)

- Visualização Zoomable - mostra os nós nos níveis inferiores (filhos) aninhados dentro do nó pai e permite realizar a ampliação desses nós para entrar e sair de cada um deles (figura 3). É uma categoria semelhante à árvore de links e nós, mas, como dito anteriormente, os pesquisadores salientaram a funcionalidade das técnicas de visualização sendo essa categoria baseada na aproximação (zoomable), diferente do recurso de expansão e retração da anterior. Técnicas e/ou ferramentas aplicadas à ontologia: CropCircles e Jambalaya. 


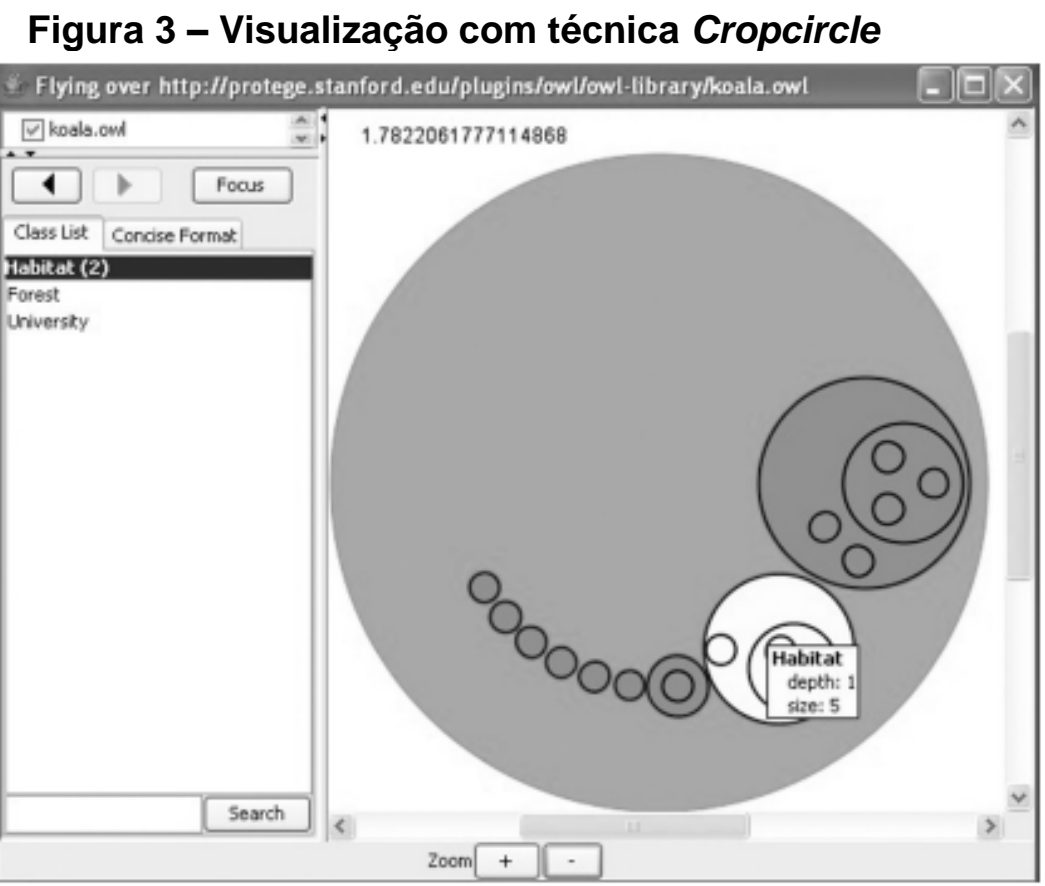

Fonte: Katifori et al. (2007)

- Preenchimento de espaço - são técnicas cuja principal característica é a utilização de todo espaço da tela subdividindo o espaço em nós e filhos. O tamanho de cada subdivisão corresponde a uma propriedade do nó atribuído a ela, como tamanho e número de nós (figura 4). Técnicas e/ou ferramentas aplicadas à ontologia: TreeMaps.

\section{Figura 4 - Visualização de Treemaps na categoria de preenchimento de espaço}

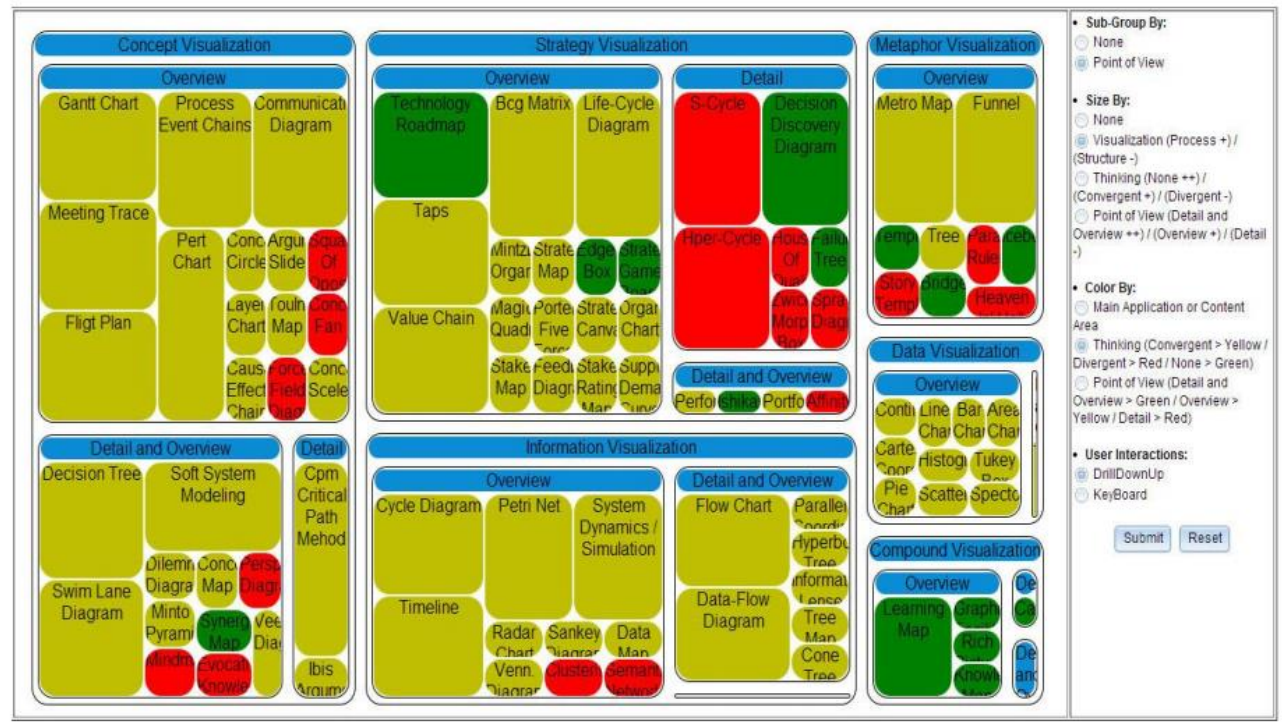

Fonte: Oliveira (2015) 
- Foco e contexto - são técnicas que distorcem a visualização para combinar contexto e foco ao exibir o nó em foco ampliado e o resto dos nós é colocado a seu redor preservando o contexto da informação apresentada (figura 5). Técnicas e/ou ferramentas aplicadas à ontologia: HyperTree Visualization, OntoRama, TGVizTab e OZONE.

Figura 5 - Visualização TGVizTab na categoria foco e contexto

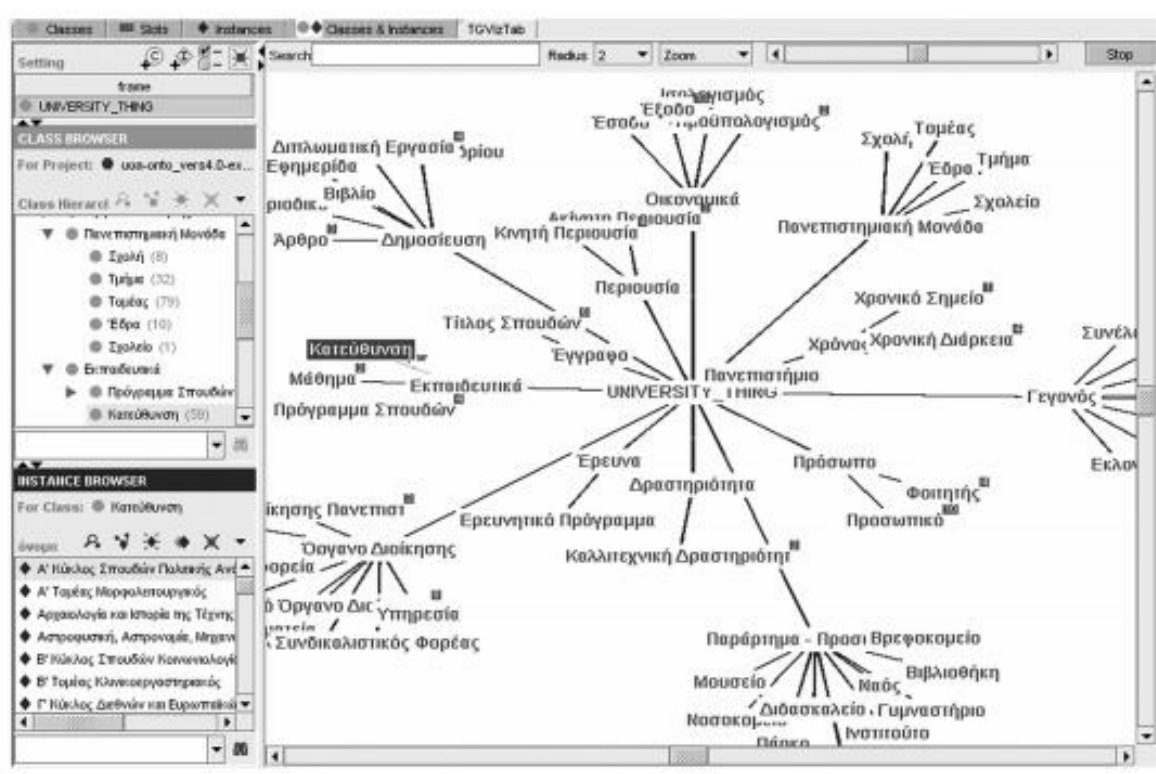

Fonte: Katifori et al. (2007)

- Paisagem 3D - é uma metáfora de paisagem que apresenta a paisagem da informação em objetos 3D agrupados através de cor e tamanho. Nessa categoria não há técnicas e/ou ferramentas aplicadas a ontologias. $\mathrm{Na}$ Figura 6 colocamos um exemplo da categoria, mas a visualização não será a representação de uma ontologia como nas categorias anteriores. 


\section{Figura 6 - Visualização em interface de paisagem 3D}

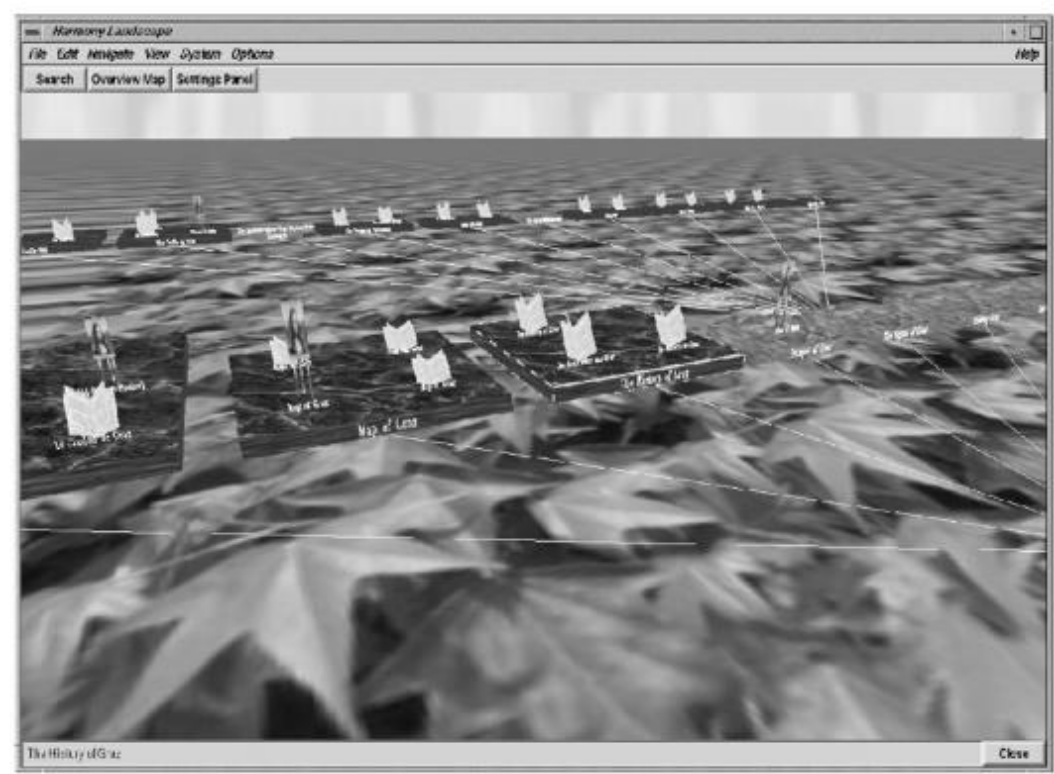

Fonte: Katifori et al. (2007)

Diante das categorias listadas e exemplificadas anteriormente fica evidente a variedade de ferramentas e modelos disponíveis para representar ontologias graficamente. Entender as diferenças entre lista identada, árvores de links e nós, visualização zoomable, preenchimento de espaço, foco mais contexto e paisagem 3D permite que o modelizador de domínio tenha um processo de escolha mais consciente e assertivo sobre qual visualização utilizar.

Como visto anteriormente, as ontologias são formadas por classes e instâncias, taxonomias, heranças, propriedades e axiomas que podem ser representados graficamente a partir das mais diversas técnicas de visualização. Dependendo da técnica ou ferramenta utilizada, um determinado elemento constituinte de uma ontologia pode se sobressair em uma visualização em detrimento de outro elemento. De modo a minimizar esse problema referente à camuflagem de características do domínio modelizado, Mendes Netto, Lima e Pierozzi Junior (2016) realizaram um estudo comparativo (quadro 1) onde buscaram identificar como cada uma das seis técnicas de visualização da informação aqui discutidas refletem os elementos formadores de ontologias. 


\section{Quadro 1 - Comparativo de representação dos modelos de visualização de ontologias}

\begin{tabular}{|c|c|c|c|c|c|}
\hline \multirow[b]{2}{*}{$\begin{array}{c}\text { Técnica de } \\
\text { visualização }\end{array}$} & \multicolumn{5}{|c|}{ Elementos das ontologias a serem representados } \\
\hline & $\begin{array}{l}\text { Classes e } \\
\text { Instâncias }\end{array}$ & Taxonomia & $\begin{array}{l}\text { Múltiplas } \\
\text { heranças }\end{array}$ & Propriedades & Axiomas \\
\hline Lista Identada & $\begin{array}{c}\text { Mostradas em } \\
\text { listas e } \\
\text { interfaces } \\
\text { separadas }\end{array}$ & $\begin{array}{l}\text { Classes } \\
\text { representadas } \\
\text { de forma } \\
\text { identada }\end{array}$ & $\begin{array}{l}\text { Replicação de } \\
\text { elementos }\end{array}$ & $\begin{array}{l}\text { Apresentadas } \\
\text { em interfaces } \\
\text { separadas }\end{array}$ & $\begin{array}{c}\text { Apresentados } \\
\text { em interfaces } \\
\text { separadas }\end{array}$ \\
\hline $\begin{array}{l}\text { Árvore de } \\
\text { links e nós }\end{array}$ & $\begin{array}{l}\text { Representados } \\
\text { por nós em } \\
\text { formas } \\
\text { geométricas }\end{array}$ & $\begin{array}{c}\text { Representada } \\
\text { por } \\
\text { proximidades } \\
\text { e ligações }\end{array}$ & $\begin{array}{l}\text { Representados } \\
\text { por ligações em } \\
\text { grafo }\end{array}$ & $\begin{array}{l}\text { Apresentadas } \\
\text { em janelas } \\
\text { separadas }\end{array}$ & $\begin{array}{c}\text { Rótulos com } \\
\text { links para } \\
\text { acesso }\end{array}$ \\
\hline Zoom & $\begin{array}{l}\text { Representados } \\
\text { em conjuntos. }\end{array}$ & $\begin{array}{l}\text { Tamanhos } \\
\text { menores e em } \\
\text { conjuntos }\end{array}$ & $\begin{array}{l}\text { Replicação de } \\
\text { elementos }\end{array}$ & $\begin{array}{l}\text { Apresentadas } \\
\text { em interfaces } \\
\text { separadas }\end{array}$ & $\begin{array}{c}\text { Apresentadas } \\
\text { em interfaces } \\
\text { separadas }\end{array}$ \\
\hline $\begin{array}{l}\text { Preenchiment } \\
\text { o de espaço }\end{array}$ & $\begin{array}{c}\text { Representados } \\
\text { por cores e } \\
\text { tamanhos } \\
\text { distintos }\end{array}$ & $\begin{array}{l}\text { Diferenciadas } \\
\text { por tamanhos } \\
\text { e cores }\end{array}$ & $\begin{array}{l}\text { Geralmente } \\
\text { não } \\
\text { representados }\end{array}$ & $\begin{array}{l}\text { Apresentadas } \\
\text { em interfaces } \\
\text { separadas }\end{array}$ & $\begin{array}{c}\text { Apresentadas } \\
\text { em interfaces } \\
\text { separadas }\end{array}$ \\
\hline $\begin{array}{l}\text { Foco e } \\
\text { contexto }\end{array}$ & $\begin{array}{l}\text { Representados } \\
\text { por Rótulos, } \\
\text { conforme foco }\end{array}$ & $\begin{array}{l}\text { Distância e } \\
\text { ligações entre } \\
\text { as classes e } \\
\text { instâncias. }\end{array}$ & $\begin{array}{l}\text { Replicação de } \\
\text { elementos }\end{array}$ & $\begin{array}{l}\text { Apresentadas } \\
\text { em interfaces } \\
\text { separadas }\end{array}$ & $\begin{array}{c}\text { Apresentadas } \\
\text { em interfaces } \\
\text { separadas }\end{array}$ \\
\hline Paisagem 3D & $\begin{array}{l}\text { Distribuídos } \\
\text { graficamente } \\
\text { em relevos. }\end{array}$ & $\begin{array}{l}\text { Linhas que } \\
\text { conectam as } \\
\text { classes }\end{array}$ & $\begin{array}{l}\text { Replicação de } \\
\text { elementos }\end{array}$ & $\begin{array}{l}\text { Apresentadas } \\
\text { em interfaces } \\
\text { separadas }\end{array}$ & $\begin{array}{c}\text { Apresentadas } \\
\text { em interfaces } \\
\text { separadas }\end{array}$ \\
\hline
\end{tabular}

Fonte: Mendes Netto, Lima e Pierozzi Junior (2016)

Entender o contexto de aplicação das visualizações, das principais características de um domínio em sua modelização e dos usuários que farão uso da ferramenta visual é fundamental para a escolha de uma representação gráfica eficaz para seu utilizador.

Diante da variedade de técnicas de visualização, tipos de interação e ferramentas para construir representações de ontologias, é imprescindível que o modelizador da estrutura conceitual e gráfica tenha consciência dos dispositivos a seu dispor e faça a escolha do recurso que melhor se aplica ao recorte do conhecimento tratado. 


\section{CONSIDERAÇÕES FINAIS}

As representações gráficas auxiliam o usuário a ter um melhor acesso à informação à medida que exemplificam conceitos e suas relações que muitas vezes podem soar abstratos. Utilizar esse recurso visual com ontologias melhora o entendimento e a absorção do conhecimento registrado e estruturado ao fazer uso da percepção visual humana que auxilia a descoberta de padrões, tendências e lacunas em um montante informacional.

Utilizamos autores seminais para entender melhor como a visualização se manifesta no contexto das ontologias. A partir da análise de trinta e quatro ferramentas e técnicas para chegar às seis categorias aqui elencadas, evidenciamos a inexistência de uma técnica apropriada a todos os cenários onde uma visualização de ontologia pode estar inserida. Além disso, pudemos perceber a variedade de ferramentas utilizadas na formulação das visualizações e quais técnicas representam melhor os elementos constituintes de uma ontologia.

Acreditamos que a compreensão e experimentação dessas ferramentas seja fundamental para desenvolver novos estudos que nos permitirão vislumbrar e projetar novos estudos acerca de visualizações que envolvam a representação de ontologias como também para fundamentar a escolha de determinada técnica para traduzir uma dada circunstância ou caraterística do conhecimento.

\section{REFERÊNCIAS}

CAMPOS, M. L. de A. Modelização de domínios de conhecimento: uma investigação de princípios fundamentais. Ciência da Informação, Brasília, v. 33, n. 1, p. 22-32, jan./abr. 2004.

CAMPOS, M. L. de A. O papel das definições na pesquisa em ontologia. Perspectivas em Ciência da Informação, Belo Horizonte, v. 15, n. 1, p. 220 238, jan./abr. 2010.

CARD, S. K.; MACKINLAY, J. D.; SHNEIDERMAN, B. Readings in information visualization: using vision to think. San Francisco: Morgan Kaufmann, 1999. 686 p. 
CARD, S. K.; MACKINLAY, J. The structure of the information visualization designs pace. In: IEEE SYMPOSIUM ON INFORMATION VISUALIZATION, 3., 1997, Phoenix. Proceedings [...]. Washington, DC: IEEE, 1997.

CHEN, C.; HÄRDLE, W.; UNWIN, A. (ed.). Handbook of data visualization. Heidelberg: Springer-Verlag, 2008.

DIAS, M. F.; CARVALHO, J. O. F. A visualização da informação e a sua contribuição para a Ciência da Informação. DataGramaZero - Revista de Ciência da Informação, v. 8, n. 5, out. 2007.

FREITAS, C. M. D. S.; CHUBACHI, O. M.; LUZZARDI, P. R. G.; CAVA, R. A. Introdução à visualização de informações. RITA: Revista de Informática Teórica e Aplicada, Porto Alegre, v. 8, n. 2, p. 143-158, out. 2001.

GRUBER, T. R. Toward principles for the design of ontologies used for knowledge sharing?. International journal of human-computer studies, v. 43, n. 5-6, p. 907-928, 1995.

KATIFORI, A.; HALATSIS, C.; LEPOURAS, G.; VASSILAKIS, C.; GIANNOPOULOU, E. Ontology visualization methods: a survey. ACM Computing Surveys, v. 39, n. 4, p. 10, 2007.

KERREN, A.; STASKO, J. T.; FEKETE, J.-D.; NORTH, C. (ed.). Information visualization: human-centered issues and perspectives. Berlim: SpringerVerlag Berlin Heidelberg, 2008.

MARCONDES, C. H.; CAMPOS, M. L. de A. Ontologia e Web Semântica: o espaço da pesquisa em Ciência da Informação. PontodeAcesso, v. 2, n. 1, p. 107-136, 2008.

MAZZA, R. Introduction to information visualization. Londres: SpringerVerlag, 2009.

MENDES NETTO, C.; LIMA, G. A. B. O.; PIEROZZI JUNIOR, I. Modelos de visualização de informação para ontologias de domínio. In: ENCONTRO NACIONAL DE PESQUISA E PÓS-GRADUAÇÃO EM CIÊNCIA DA INFORMAÇÃO (ENANCIB), 17., 2016, Salvador. Anais [...]. Salvador: UFBA, 2016.

MENDES NETTO, C.; LIMA, G. Visualização de Ontologias: estudos e perspectivas. Informação \& Sociedade: Estudos, João Pessoa, v. 27, n. 3, p. 59-72, set./dez. 2017.

MENDES NETTO, C. Proposta de modelo de requisitos para ferramentas de visualização de ontologia de domínio. 2017. 160 f. Tese (Doutorado em Gestão e Organização do Conhecimento) - Universidade Federal de Minas Gerais, Belo Horizonte, 2017. 
NASCIMENTO, H. A. D.; FERREIRA, C. B. R. Visualização de informações: uma abordagem prática. In: CONGRESSO DA SOCIEDADE BRASILEIRA DE COMPUTAÇÃO, 25., 2005, São Leopoldo. Anais [...]. São Leopoldo:

UNISINOS, 2005. p. 1262-1312.

OLIVEIRA, E. C. Uma proposta de metamodelo de visualização da informação, baseada em treemaps. 2015. 102 f. Dissertação (Mestrado em Ciências) - Faculdade de Engenharia Elétrica, Universidade Federal de Uberlândia, Uberlândia, 2015.

SHNEIDERMAN, B. The eyes have it: a task by data type taxonomy for information visualizations. In: SYMPOSIUM ON VISUAL LANGUAGENS,1996, Boulder. Proceedings [...]. Boulder: IEEE, 1996.

VIEIRA, J. M. L.; PINHO, F. A. A contribuição da organização e da visualização da informação para os sistemas de recuperação de informação. Informação \& Informação, Londrina, v. 20, n. 1, p. 110-136, jan./abr. 2015.

WARE, C. Information visualization: perception for design. 2. Ed. New York: Morgan Kaufmann Publishers, 2004.

ZHANG, J. Visualization for information retrieval. New York: Springer, 2008. $287 \mathrm{p}$.

\title{
ONTOLOGY VISUALIZATION: PERSPECTIVES FOR THE REPRESENTATION OF MODELING DOMAIN
}

\begin{abstract}
Objective: It aims to identify the information visualization techniques suitable to represent this Knowledge Organization System. Methodology: It has as methodology a qualitative approach, of applied nature and exploratory objective where a bibliographical study was carried out. Results: It presents and exemplifies the techniques and tools identified list, trees of links and nodes, zoomable visualization, space filling, focus plus context and 3D landscape. Conclusions: It points out the inexistence of an appropriate technique for all the scenarios where an ontology visualization can be inserted.
\end{abstract}

Descriptors: Ontologies. Information visualization (contribution). Ontology visualization.

\section{VISUALIZACIÓN DE ONTOLOGÍAS: PERSPECTIVAS PARA LA REPRESENTACIÓN DE DOMINIOS MODELIZADOS}




\section{RESUMEN}

Objetivo: Su propósito es identificar técnicas de visualización de información capaces de representar este Sistema de Organización del Conocimiento. Metodología: es un enfoque cualitativo, de carácter aplicado y objetivo exploratorio donde se realizó un estudio bibliográfico. Resultados: ejemplifica técnicas y herramientas de listas con sangría, árboles de enlaces y nodos, visualización ampliable, relleno de espacios, enfoque más contexto y paisaje 3D. Conclusiones: señala la inexistencia de una técnica adecuada para todos los escenarios donde se puede insertar una visualización de ontología.

Descriptores: Ontologías. Visualización de información (contribución). Visualización de ontologías.

Recebido em: 06.12.2021

Aceito em: 13.12.2021 